\title{
Negative behaviours as the reason for referral to a liaison old age psychiatrist
}

\section{R. Euba}

Consultant and Senior Lecturer in Old Age Psychiatry, Guy's, King's and St. Thomas' Schools of Medicine, London.

UNITED KINGDOM

\begin{abstract}
The analysis of 130 consecutive referrals to a liaison old age psychiatrist in two different London hospitals revealed that nearly half of these requests mentioned negative behaviours as the reason for referral. The common factor between these behaviours was that they made the delivery of the treatment or discharge plan very difficult. This type of referral was more common for patients who had stayed in hospital for longer at the time of the request.
\end{abstract}

\section{Introduction}

The benefits of a fluid psychiatry liaison service for older patients are widely recognised. It is also well known that the characteristics of liaison referrals for this age group tend to differ from those of younger patients (Cardoso et al. 1994, Clarke et al. 1995, Krakowski 1979, Levitte and Thornby 1989). Older patients who are referred to the psychiatrist are more likely to have an organic syndrome, and the consultation is less likely to be the result of a self-harm episode. Other issues, such as the presence of multiple pathologies, assessment of competency, or the aftercare and placement in the community of patients with dementia, further define liaison work in old age psychiatry. The aim of this study was to assess a series of liaison referrals to an old age psychiatrist and to estimate the proportion of these referrals that mentioned a negative behaviour -that would hinder the delivery of the management plan- as the main reason for referral.

\section{Methods}

A series of 130 consecutive written liaison referrals to two different psychogeriatric services, each in a different district hospital in East London, England, was examined. The 
author had assessed each of these patients over a period of two years. Both hospitals in the study used the same referral protocol, which consisted of a standard referral form, in which the referrer completed the required information. This included the referrer's name and ward, the demographic details of the patient, the reason for their admission to hospital, their current medical or surgical status, their medication regime, and the reason for the referral to the psychogeriatric service, expressed in an open format and in the words of the referrer. The form did not prompt the referrer with a list of possible reasons for referral. All referrals came from physicians and surgeons based in acute medical and surgical wards. Virtually all referrals indicated that the patient had symptoms such as confusion, low mood, or abnormal behaviour, that would suggest the presence of a significant psychiatric disorder, but the psychiatric assessor later felt, after examining the patients, that $19 \%$ did not merit a psychiatric diagnosis. When a request for consultation mentioned one of the following as the main reason for the referral: hostility, refusing to eat, not accepting treatment, lack of cooperation with physiotherapy, requesting discharge against medical advice, factitious complaints, or refusing to have institutional care after discharge -when this had been judged to be necessary by the treating physician-, the referral was classified by the author as "Negative Behaviours Impeding Implementation of Management Plan". When a request for consultation mentioned any other reason for referral, but none of those described above, the referral was excluded from this category. Other reasons for referral included: the need to assess a patient's depression or suicidality following a self-harm episode, assessment of an apparent psychosis, assessment of confusion, advice regarding psychotropic medication, and assessment of mental capacity (not related to a disagreement about the treatment or discharge plan).

\section{Results}

Of the 130 patients, $55 \%$ were female and the average age was 80 years $(95 \%$ confidence interval: 79 to 82 ); the average age for males and females was 79 and 82 respectively. $64 \%$ lived alone. $42 \%$ had been seen by a psychiatrist before.

The mean time interval since admission to hospital until the request for a psychiatric assessment was 27 days (95\% confidence interval: 21 to 32 days). 49 cases were referred for assessment of an apparent organic disorder, 55 because of a functional disorder, and the remaining 26 for other reasons. 55 referrals $(42 \%)$ fell into the category of "Negative Behaviours Impeding Implementation of Management Plan" (Table I). Females were more likely to be included in this category than males (Odds Ratio=2.3;

Table I

Negative behaviours impeding the implementation of the management plan

$\mathrm{n}=55(\%)$

Hostile behaviour

Refusing institutional care after discharge

$19(34.5)$

$14(25.5)$

$9(16.4)$

$6(10.9)$

$5(9.1)$

$1(1.8)$

$1(1.8)$ 
95\% confidence interval: 1.1 to 4.8$)$. Patients who were confused at the time of their assessment were referred after spending an average of 31 days in hospital, whereas nonconfused patients were referred after an average of 18 days. This difference was statistically significant: $p=0.004$. However, the length of time from admission to referral did not correlate with age. There was no association between belonging to the "negative behaviours" group and age, whether the patient lived alone or not, had been seen by a psychiatrist before or not, whether or not the patient had received a psychiatric diagnosis after specialist examination, or whether the consultation had been requested for assessment of an organic or a functional disorder. The levels of staffing in the different wards at the time of each referral was not recorded, but both hospitals in the study produced a similar proportion of referrals that were subsequently included in the "negative behaviours" group, and no individual wards or referring doctors produced a significant excess of referrals later included in that group. After dividing the period of time in days lapsed between admission to hospital and psychiatric referral into three percentiles, it was found that a longer time was associated with an increased likelihood of being included into the "Negative Behaviours Impeding Implementation of Management Plan" category: Mantel-Haenszel test for linear association $\chi^{2}=5.3$, degrees of freedom $=1, \mathrm{p}=0.021$.

\section{Discussion}

Referring doctors in this small study had experienced difficulties in delivering their management plan to nearly half of all elderly hospital patients eventually referred for psy- chiatric consultation. This percentage suggests that this is likely to be a significant factor in determining which cases will be seen by the liaison psychogeriatrician. The characteristics of this sample are comparable to samples in previous studies (Loane and Jefferys 1998), although the female bias was smaller than usual. The relative excess of females in the "Negative Behaviours" group was small and possibly artefactual. The levels of staffing in the ward at the time of the referral were not recorded in this study. It is possible that lower levels of staffing at a given time might have resulted in an increased difficulty in the ability to implement a management plan. This is a relevant area for future research. The correlation between length of stay until psychiatric referral and likelihood of belonging to the "Negative Behaviours" group was an interesting finding; displaying negative behaviours that impede the implementation of the management plan, including discharge arrangements, would naturally result in an extended length of stay in hospital, thus making a late referral possible. In fact, one of the largest subgroups in this category consisted of patients with dementia refusing institutional care after discharge, but who were considered by the referring doctor to be unable to continue living independently. In most of these cases, the psychiatrist was asked to give his opinion on the patient's mental competency to choose a place of residence. This would be consistent with the findings of a Dutch study which concluded that late referrals were associated with high social vulnerability (De Jonge et al. 2000). On the other hand, it seems plausible that many of the requests for psychiatric consultations for patients who disagreed with their treatment plan were delayed precisely because the referring doctor had already spent some time already trying to deliver his or her management plan unsuccessfully. It must be emphasised that 
the majority of the patients in this series had a psychiatric disorder, and that their negative behaviours, such as refusing to eat or being hostile to staff, were often manifestations of those disorders. It follows therefore that the referrals made to address these behaviours were totally appropriate. Similarly, when a disagreement about treatment between the patient and the treating doctor arose in the context of an apparently diminished mental capacity, the request of a psychiatric opinion was a legitimate course of action. There are of course many complex issues regarding consent to treatment and the relationship between doctor and patient, as well as the interaction between timing of referral and length of stay (Ormont et al. 1997, Handrinos et al. 1998), but the discussion of such questions is beyond the scope of this report. Finally, it is this author's opinion that seeking the help of a psychiatrist for the successful delivery of a medical, surgical, or social management plan is also a very legitimate, but poorly recognised, reason for referral.

\section{References:}

Cardoso G, Ismail F, Barbosa A. O idoso no hospital geral e a psiquiatría de ligaçao. Acta Med Port 1994; 7 : 601-605.
Clarke DM, McKenzie DP, Smith GC: The recognition of depression in patients referred to a consultation-liaison service. J Psychosom Res 1995; 39: 327-334.

De Jonge P, Huyse-Frits J, Ruinemans G, Stiefel FC, Lyons JS, Slaets JPJ. Timing of psychiatric consultations: The impact of social vulnerability and level of psychiatric dysfunction. Psychosomatics 2000; 41: 505-511.

Handrinos D, McKenzie D, Smith-Graeme C. Timing of referral to a consultation-liaison psychiatric unit. Psychosomatics 1998; 39: 311-317.

Krakowski AJ. Psychiatric consultation for the geriatric population in the general hospital. Bibl Psychiatr 1979; 159: $163-185$.

Levitte SS, Thornby JI. Geriatric and nongeriatric psychiatry consultation: a comparison study. Gen Hosp Psychiatry 1989; 11: 339-344.

Loane R, Jefferys P. Consultation-liaison in an old age psychiatry service. Psychiatr Bull R Coll Psychiatr 1998; 22: 217-220.

Ormont MA, Weisman HW, Heller SS, Najara JE, Shindledecker RD. The timing of psychiatric consultation requests: Utilization, liaison, and diagnostic considerations. Psychosomatics 1997; 38: 38-44.

Address for correspondence:

Memorial Hospital, Shooters' Hill,

London SE18 3RZ,

UNITED KINGDOM

e-mail: Rafael.Euba@oxleas.nhs.uk 